\title{
Parking Spaces Detection Based on Fingerprint Algorithm
}

\author{
Kunshan Xing ${ }^{1,}$, Zhigang Wen ${ }^{2, b}$ \\ ${ }^{1}$ School of Electronic Engineering, Beijing University of Posts and Telecommunications, Beijing, \\ China \\ ${ }^{2}$ Institute of Sensing Technology and Business, BUPT, Wuxi, China \\ aemail: ksxing@126.com, bemail: zwen@bupt.edu.cn
}

\section{Keywords: Fingerprint Algorithm; Parking Spaces Detection; Internet of things}

\begin{abstract}
With the development of society, the number of private cars is increasing year by year, leading to parking management become an important issue of nowadays social traffic. The traditional method of manual management of the parking lot cannot meet requirements of large-scale parking lots. Because of the above problems, smart parking lot based on Internet of things is getting more and more attention. The main research of this paper is the real-time detection of parking spaces in smart parking lots, establishing a parking spaces perception system and using fingerprint algorithm to detect the accurate occupancy situation of parking spaces.
\end{abstract}

\section{Introduction}

Deploying Internet of things labels as perception nodes in the parking spaces, receivers can receive signal from perception nodes, and occupancy situation of parking spaces in parking lots can be automatically perceived. Data of perception result unload to the server, and parking spaces occupancy detection is almost real time. A lot of artificial intervention after one-time deployment is unnecessary, resource can be conserved, and quality of parking experience can be effectively improved.

Parking spaces perception system mainly includes three parts: the perceptual nodes, receivers and database processing. Perception nodes are deployed in the smart parking lot; periodically send signals to the receivers and time interval can be set by mangers. Receivers receive signal from perceptual nodes and transmit data to the server through the gateway. Construct the fingerprint database on the server with the data obtained from transmission .Database processing analyzes the data on a computer using certain fingerprint algorithm and obtain calculation results. This paper mainly focuses on the part of fingerprint algorithm, discussing several common fingerprint algorithms to achieve the accurate judgment of parking space occupancy state and comparing performance of several algorithms.

\section{Design of Fingerprint Algorithm}

Current fingerprint algorithm [1] [2] is mainly used in positioning service. Fingerprint positioning algorithm can be divided into parts [3]: off-line training and on-line positioning. Offline part takes record of each reference node signal characteristic parameters in different coordinate points as fingerprint, signal characteristic parameters can be various, such as signal strength, multipath power phase angle and so on. A piece of fingerprint corresponds to a coordinate point, using these data to establish the fingerprint library. Online positioning part takes a real-time received signal to match the fingerprint from the fingerprint library by certain algorithm, and calculates the position coordinates of current position.

The advantages of the positioning fingerprint algorithm are as follows: high positioning accuracy, one base station can realize the positioning process, making full use of existing facilities, do not need to change the hardware of devices and the system needs only very few extra equipment, update and maintenance takes small effects on users. However, the downside of the positioning fingerprint algorithm includes the early stage of the workload is very big, and not suitable for 
regional environmental which change too fast. Fingerprint positioning accuracy depends on the size of the database, to improve the measurement precision needs the detailed measurements of locate area, and establish a huge database. The database must be regularly or not regularly updated, no-data area will not be able to provide positioning services.

This paper takes the idea of fingerprint positioning algorithm to parking space detection. Parking space detection fingerprint algorithm can be divided into two stages like above: off-line training and on-line detection. First stage, offline training records signal strength from receivers to build a fingerprint database. Second stage, online part will take a current received signal strength value to match with each piece of fingerprint in the database, according to the calculation can obtain a state value to judge the real-time parking space occupancy state.

\section{Parking Detection Algorithm Design}

Parking space detection fingerprint algorithm can be divided into two stages like above: off-line training and on-line detection. The first step is off-line training; the main task of this step is to establish the fingerprint database. Assuming two states values: 1 and 0 to represent two different states, namely occupied state and empty state. Each state records numbers of group signal strength value, using a type of vector expressed as $\mathrm{RSSI}=\left(\mathrm{RSS}_{1}, \mathrm{RSS}_{2}, \ldots, \mathrm{RSS}_{\mathrm{n}}\right)$ to establish a fingerprint database [4], $n$ represents the number of receivers that a parking space label corresponds to. The second step is on-line detection, the parking space label receive signal values from receivers , using a vector expressed as $S=\left(S_{1}, S_{2}, \ldots, S_{n}\right)$, $n$ also represents the number of receivers that a parking space label corresponds to. Then, taking vector $\mathrm{S}$ to match with each fingerprint from fingerprint database by certain fingerprint algorithm, selecting the minimum distance vector and its state value, thinking vector $\mathrm{S}$ corresponds to the same state value. According to this state value, the occupancy situation can be judged. The process of fingerprint algorithm is described as figure 1:

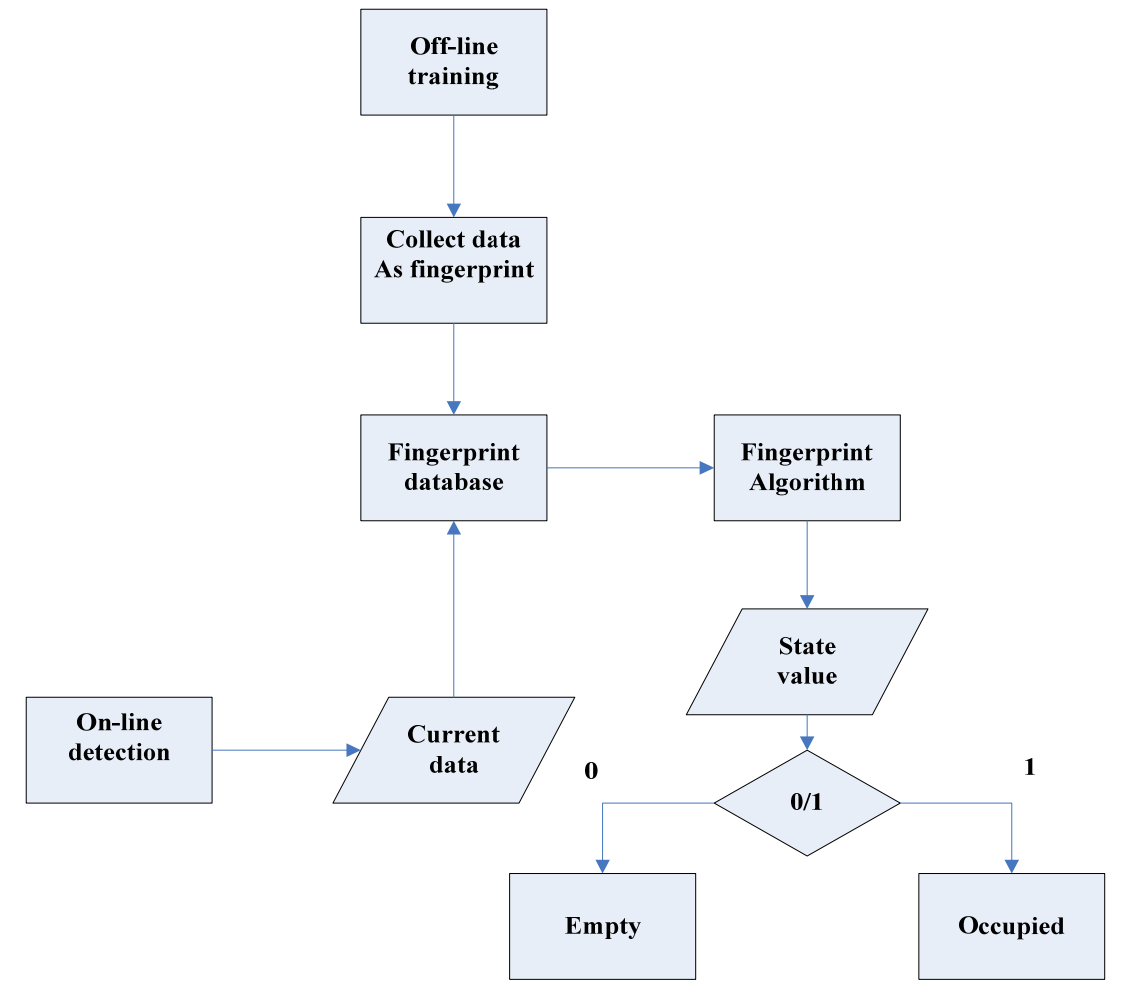

Figure 1. The process of fingerprint algorithm

This paper uses two types of deterministic algorithms and probability algorithms as the matching algorithms. Deterministic algorithm compares similarity parameters of real-time signal and training fingerprint, choosing the most similar status as state evaluation. Deterministic fingerprint algorithm can be divided into three categories: nearest neighbor (NN) algorithm, K 
nearest neighbor (KNN) algorithm and weighted $\mathrm{K}$ nearest neighbor (WKNN) algorithm. Probability algorithm uses a method of mathematical to solve the uncertainty caused by complex environment. The most commonly algorithm is Naive Bayesian (NB) algorithm.

The most basic algorithm of fingerprint algorithm is Nearest Neighbors (NN) algorithm. First of all, receivers record signal strength as $S=\left(S_{1}, S_{2}, \ldots, S_{n}\right)$,n represents the number of receivers. After that, taking $S$ match with $F_{i}=\left(f_{1}, f_{2}, \ldots, f_{n}\right)$ from fingerprint database, calculating distance between

$\mathrm{S}$ and Fi. Distance formula is as follows:

$$
D_{i}=\left|\sum_{i=1}^{n}\left(S_{i}-f_{i}\right)\right|^{1 / 2}, i=1,2,3, \ldots, l
$$

Taking the minimum distance Di as the result. According to the result Di, we can obtain state value and regard the current as the same state with Fi.

$\mathrm{K}$ nearest neighbor (KNN) algorithm is the improved algorithm of Nearest Neighbors (NN) algorithm. Taking the average of $\mathrm{K}$ vector value as the vector value $\mathrm{S}$, then calculate distance between $S$ and $F_{i}$.

$$
S=\left(S_{1}, S_{2}, \ldots, S_{n}\right)=\frac{1}{k} \sum_{i=1}^{k}\left(S_{1 i}, S_{2 i}, \ldots . S_{n i}\right)
$$

Weighted $\mathrm{K}$ nearest neighbor (WKNN) algorithm selects the $\mathrm{K}$ vector, not to calculate their average value as measured output, but to each vector corresponding to a state of locomotive on a weighted coefficient, the sizes of the weights decided the proportion of final results. Finally, the calculation result of the output is:

$$
D_{i}=\left|\sum_{i=1}^{n} w_{i}\left(S_{i}-f_{i}\right)\right|^{1 / 2}, i=1,2,3, \ldots, l
$$

$\mathrm{W}_{\mathrm{i}}$ represents the weighted value of each receivers.

Naive Bayesian (NB) algorithm [5] based on the Bayesian formula:

$$
P(B \mid A)=\frac{P(A \mid B) P(B)}{P(A)}
$$

The foundation of naive Bayesian is: to give the certain classification, and calculate probability of each category in the conditions, which probability is the max, thought that this classification belongs to this category. Under no other information available, we will choose the biggest category of conditional probability. Algorithm steps are as follows:

Step1Aassuming that $x=\left\{a_{1}, a_{2}, \ldots, a_{m}\right\} \quad$ to be a classified item and each a is a feature of $\mathrm{x}$ attributes.

Step2.Existing a category set $C=\left\{y_{1}, y_{2}, \ldots, y_{n}\right\}$, each y is regarded as a category type

Step3.To calculate $P\left(y_{1} \mid x\right), P\left(y_{2} \mid x\right), \ldots, P\left(y_{n} \mid x\right)$

Step3. If $P\left(y_{k} \mid x\right)=\max \left\{P\left(y_{1} \mid x\right), P\left(y_{2} \mid x\right), \ldots, P\left(y_{n} \mid x\right)\right\}$, regarding $x \in y_{k}$

Now, the point of algorithm is to calculate conditional probability in step3. Use the following approach:

1) Find a set of known classification for the classification, the collection is called the training sample set.

2) If the condition of several characteristic properties are independent, then according to Bayesian' theorem is derived as follows:

3) $\quad P\left(y_{i} \mid x\right)=\frac{P\left(x \mid y_{i}\right) P\left(y_{i}\right)}{P(x)}$

4) Because the denominator for all categories is a constant value, so we just maximize molecules are available. And the conditions of each feature attribute is independent, so there are: 


$$
P\left(x \mid y_{i}\right) P\left(y_{i}\right)=P(a 1 \mid y i) P(a 2 \mid y i) \ldots P\left(a_{m} \mid y_{i}\right) P\left(y_{i}\right)=P\left(y_{i}\right) \prod_{j=1}^{m} P\left(a_{j} \mid y_{i}\right)
$$

In this paper,in order to detect parking spaces state,we regard each space is used or not as two types of state value, using number 0 represents the empty state, and number 1 represents the occupied state. First of all, receiver records signal strength as $S=\left(S_{1}, S_{2}, \ldots, S_{n}\right), n$ represents the number of receivers. Calculating $\max \left\{\mathrm{P}\left(\mathrm{C}_{1} \mid \mathrm{S}\right), \mathrm{P}\left(\mathrm{C}_{2} \mid \mathrm{S}\right)\right\}$, if state value is 0 means the space is empty, else means the space is occupied.

\section{Test results}

\section{Deterministic algorithm}

In this test, three receivers record RSS value of a label at the same time. Off-line training using average value of 30 group receive signal strength $\mathrm{RSSI}=\left(\mathrm{RSSI}_{1}, \mathrm{RSSI}_{2}, \mathrm{RSSI}_{3}\right)$ as fingerprint to build a fingerprint database. On-line detection part, receivers receive signal strength value as $S=\left(S_{1}\right.$, $\mathrm{S}_{2}, \mathrm{~S}_{3}$ ).Matching $\mathrm{S}$ with each fingerprint from database, recording to the result to obtain the real-time state value.

Table 1. The experimental results of deterministic algorithms

\begin{tabular}{|l|l|l|l|l|}
\hline Algorithm & Parameter & $\begin{array}{l}\text { Number of } \\
\text { test }\end{array}$ & $\begin{array}{l}\text { Number of } \\
\text { mistakes }\end{array}$ & $\begin{array}{l}\text { Correct } \\
\text { proportion }\end{array}$ \\
\hline NN & & 50 & 18 & $64 \%$ \\
\hline \multirow{2}{*}{ KNN } & $\mathrm{K}=2$ & 50 & 13 & $72 \%$ \\
\cline { 2 - 5 } & $\mathrm{K}=3$ & 50 & 12 & $74 \%$ \\
\hline \multirow{2}{*}{ WKNN } & $\begin{array}{l}\mathrm{K}=2, \mathrm{w} 1=0.25, \\
\mathrm{w} 2=0.5, \mathrm{w} 3=0.25\end{array}$ & 50 & 8 & $84 \%$ \\
\hline
\end{tabular}

\section{Probability algorithm}

In this test, the result will be different when the number of receivers changes. We take number of receiver as one, two and three to calculate joint probability. Assuming receivers are independent of each other, signal characteristics to obey Gaussian distribution.

$$
P\left(S_{i} \mid C\right)=\frac{1}{\sqrt{2 \pi} \sigma_{C}} \exp \left(-\frac{\left(S_{i}-\mu_{c}\right)^{2}}{2 \sigma_{c}^{2}}\right)
$$

$\mu$ is the average value, $\sigma$ is the standard deviation.

Gaussian distribution can be seen as figure 2 .

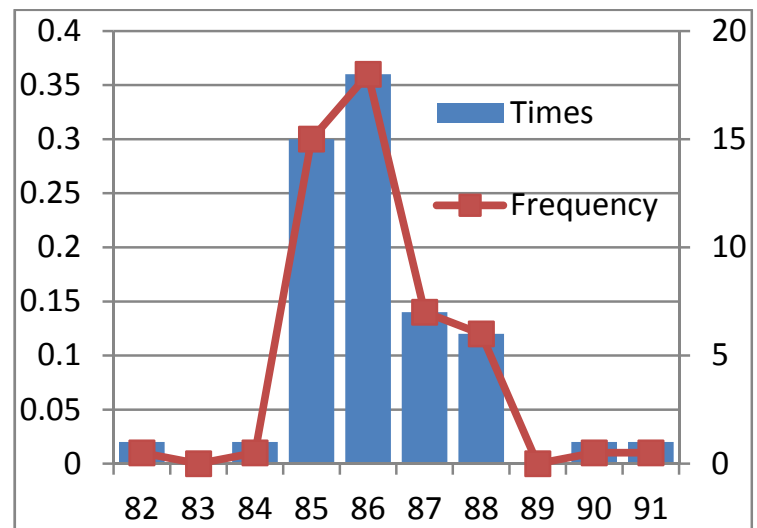

(a) Times and frequency

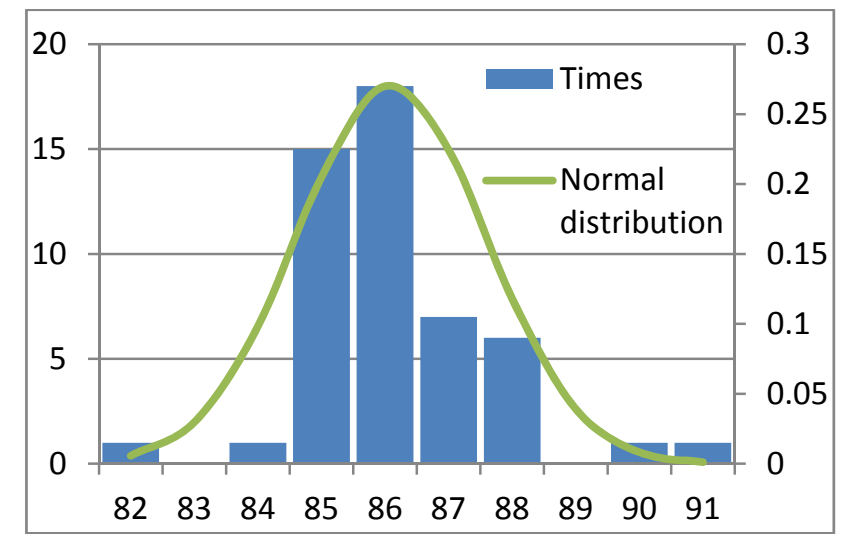

(b) times and normal distribution

Figure 2. Gaussian distribution

$\mathrm{C}$ represents the state value, $\mathrm{C}=0$ is empty state, $\mathrm{C}=1$ is occupied state. $\mathrm{n}$ represents the number of receivers.

Because $P\left(\prod_{i=1}^{n} S_{i}\right)$ is a constant value, and $P(C=0)=P(C=1)=0.5$, formula can be changed to:

$\mathrm{P}\left(\mathrm{C} \mid \prod_{\mathrm{i}=1}^{\mathrm{n}} \mathrm{S}_{\mathrm{i}}\right)=\mathrm{P}\left(\mathrm{S}_{1} \mid \mathrm{C}\right) \mathrm{P}\left(\mathrm{S}_{2} \mid \mathrm{C}\right) \ldots \mathrm{P}\left(\mathrm{S}_{\mathrm{n}} \mid \mathrm{C}\right)$

So we regard that if $\mathrm{P}\left(\mathrm{C}=0 \mid \prod_{\mathrm{i}=1}^{\mathrm{n}} \mathrm{S}_{\mathrm{i}}\right)>\mathrm{P}\left(\mathrm{C}=1 \mid \prod_{\mathrm{i}=1}^{\mathrm{n}} \mathrm{S}_{\mathrm{i}}\right)$, the space is empty, else if $\mathrm{P}\left(\mathrm{C}=1 \mid \prod_{\mathrm{i}=1}^{\mathrm{n}} \mathrm{S}_{\mathrm{i}}\right)>$ 
$\mathrm{P}\left(\mathrm{C}=0 \mid \prod_{\mathrm{i}=1}^{\mathrm{n}} \mathrm{S}_{\mathrm{i}}\right)$, the space is occupied. Test result as follows:

Table 2. The experimental results of probability algorithms

\begin{tabular}{|l|l|l|l|l|}
\hline Algorithm & $\begin{array}{l}\text { Number of } \\
\text { receivers }\end{array}$ & $\begin{array}{l}\text { Number of } \\
\text { test }\end{array}$ & $\begin{array}{l}\text { Number of } \\
\text { mistakes }\end{array}$ & $\begin{array}{l}\text { Correct } \\
\text { proportion }\end{array}$ \\
\hline NB & 1 & 50 & 9 & $82 \%$ \\
\cline { 2 - 5 } & 2 & 50 & 2 & $96 \%$ \\
\cline { 2 - 5 } & 3 & 50 & 1 & $98 \%$ \\
\hline
\end{tabular}

Through table 2 we can see that probability algorithms can obtain a high correct proportion result for parking detection. In addition, correct proportion increases with the number of receivers, but number of receivers also influence the experimental workload. The more the number of receivers, the more work of construction of the fingerprint database, the more time it works.

\section{Conclusion}

In order to detect state of parking spaces, this paper use fingerprint algorithm to correctly judge occupy situation of spaces. Deterministic algorithms and probability algorithms are used to this research. Deterministic algorithm has less computation and more simple. Probability algorithm is more accurate but more computationally. According to the experimental results, we can get a higher correction proportion result than traditional methods. To sum up, the fingerprint algorithm enhances the accuracy in parking space detection effectively.

\section{Acknowledgments}

The work presented in this paper was supported by the National Great Science Specific Project (Grants No. 2014ZX03002002-004) and National Natural Science Foundation of China (Grants No. NSFC-61471067).

\section{References}

[1] He Qiang. Research and implementation on ZigBee-based RSS fingerprint indoor positioning methods.[J], 2009.

[2] Wang Sai-wei .Research on WLAN indoor location method based on fingerprint. [D].Harbin. Harbin Institute University, 2009:16-30

[3] Wang Zhongmin. Improved fingerprint algorithm for smart phone indoor positioning.[J].Xi'an. Journal of Xi'an University of Posts and Telecommunications,2014.

[4] Rong Xiao-feng, YANG Na. Location error analysis and simulation of location fingerprint technology based on RSS.[J].Xi'an. Journal of Xi'an University of Posts and Telecommunications, 2010

[5] Chen Tie-jun, Jing Feng-nian, Duan Yi-hai. RSSI-based Bayesian anti-spam filtering algorithm. [J].Computer Engineering and Design, 2015 